\title{
INDUCTION OF CELL-MEDIATED IMMUNE RESPONSES AFTER VACCINATION WITH INACTIVATED OIL-EMULSION NOCARDIA SERIOLAE IN AMBERJACK SERIOLA DUMERILI
}

\author{
Kyosuke Araki ${ }^{1 *}$, Megumi Matsumoto ${ }^{2}$, Kazuma Hayashi ${ }^{1}$, Atsushi Yamamoto ${ }^{2}$ \\ ${ }^{1}$ Faculty of Fisheries, Kagoshima University, 4-50-20 Shimoarata, Kagoshima, 890-0056, Japan \\ ${ }^{2}$ The United Graduate School of Agricultural Sciences, Kagoshima University, 1-21-24 Korimoto, \\ Kagoshima, 890-8580, Japan
}

Nocrdia seriolae is a Gram-positive intracellular bacterium that causes Nocardiosis in cultured yellowtail Seriola quinqueradiata and amberjack Seriola dumerili, which results in serious economic losses in aquaculture production in Japan. Therefore, an effective vaccine is urgently needed. In mammlals, cell-mediated immunity (CMI) plays crucial role in protection from intracellular pathogen infection. In the present study, induction of cell-mediated immune response was evaluated in amberjack injected with formalin-killed $N$. seriolae vaccine containing oil adjuvant. Fish were sensitized with $N$. seriolae FKC alone or oil-emulsion of FKC (O-FKC) at $10^{8}$ CFU/fish by i.p. injection. After 8 months, fish were challenged by i.p. injection with $10^{6} \mathrm{CFU} /$ fish of live $N$. seriolae. Agglutination titer in FKC-vaccinated fish showed high values $\left(2^{5}\right.$ to $\left.2^{7}\right)$ whereas those in O-FKC injected fish wewe less than $2^{3}$ at Day3 and Day7 post-challenge. In addition, gene expression level of $I F N \gamma$ was up-regulated only in O-FKC injected fish at Day3 post-infection. Moreover, although Th2-skewing condition was confirmed in FKC-vaccinated fish, O-FKC vaccination induced predominance of Th1 but not Th2 based on gene expression analysis of T-bet/Gata-3 at Day 7 post-challenge. Relative percent survivals of FKC- and O-FKC-vaccinated fish were $26.9 \%$ and $43.6 \%$, respectively at Day 9. These results suggest that inactivated oil-emulsion $N$. seriolae vaccine exhibit CMI-inducing effect in fish.

\section{KEYWORDS}

Cell-mediated immunity, Vaccine, Intracellular pathogen, Oil adjuvant, Th1/Th2, IFN $\gamma$

*Corresponding author. Tel.:+81 992864162; Fax: +81 992864162

E-mail address: araki@fish.kagoshima-u.ac.jp 